\title{
Unmet Needs in the Management of Neovascular Age- Related Macular Degeneration (nAMD): Clinician's Perspective
}

\author{
Nishikant Borse ${ }^{1}$, Rahul Surti ${ }^{2}$, Nitin Maksane ${ }^{3}$, Maulik Bhavsar ${ }^{3,}$,, Manan Thaker $^{3}$ \\ ${ }^{1}$ Insight Eye Clinic, Mumbai, India \\ ${ }^{2}$ Krishna Eye Centre, Mumbai, India \\ ${ }^{3}$ Novartis Healthcare Pvt. Ltd., Mumbai, India
}

Email address:

insighteyeclinics@gmail.com (N. Borse),drrahul.surti@gmail.com (R. Surti),nitin.maksane@novartis.com (N. Maksane), maulik.bhavsar@novartis.com (M. Bhavsar),manan.thaker@novartis.com (M. Thaker)

${ }^{*}$ Corresponding author

\section{To cite this article:}

Nishikant Borse, Rahul Surti, Nitin Maksane, Maulik Bhavsar, Manan Thaker. Unmet Needs in the Management of Neovascular Age- Related Macular Degeneration (nAMD): Clinician's Perspective. International Journal of Ophthalmology \& Visual Science.

Vol. 5, No. 4, 2020, pp. 90-100. doi: 10.11648/j.ijovs.20200504.12

Received: September 27, 2020; Accepted: October 16, 2020; Published: November 11, 2020

\begin{abstract}
Neovascular age-related macular degeneration (nAMD) is the leading cause of severe vision loss and blindness across the globe. Aging is one of the main risk factors that can be attributed to development and progress of nAMD. This review article focuses on the various nAMD associated challenges that clinicians face with respect to its diagnosis, treatment and follow up. Challenges associated with diagnosis of nAMD include delayed diagnosis and challenges related to Optical Coherence topography (OCT) imaging. Even though anti-VEGFs are the mainstay of treatment and are effective in maintaining or improving vision, treating nAMD comes with its own set of burdens from the clinician's perspective. Clinicians are faced with the choice of different types of anti-VEGFs, treatment regimens and the chronic, variable and unpredictable nature of nAMD all of which can have a major impact on treatment outcomes. Monitoring associated with treatment is also a major burden. Newer anti-VEGFs which have a longer, sustained action may help decrease this burden. Other challenges include stressed out clinic capacities, lack of equipment and trained personnel. The aim of this review article is to highlight the challenges attributed to the diagnosis and management of nAMD from a clinician's perspective, especially important in developing countries like India which face a combination of high disease burden, lack of disease awareness, lack of facilities, equipment and personnel. Together, they can have a disastrous effect, impacting vision of the aging population. There is a dearth of India-specific data on the various challenges of diagnosis and treatment of nAMD. Such data can act as a building block upon which strategic steps can be developed and implemented which in turn may help save the vision of the huge Indian population who are afflicted by nAMD.
\end{abstract}

Keywords: Neovascular Age Related Macular Degeneration, nAMD, Anti-VEGFs, Treatment Burden, Unmet Need, Management, India

\section{Introduction}

Age-related macular degeneration (AMD) is an acquired disease of the macula and is characterized by progressive, severe and irreversible visual impairment [1, 2]. AMD usually afflicts the elderly population. The Age-related eye disease study (AREDS) classifies AMD into 3 stages: early, intermediate and advanced. Advanced AMD is characterized by geographic atrophy (GA) and neovascular maculopathy also called as neovascular AMD or nAMD and is vision threatening $[1,3]$.

In $\mathrm{GA}$, the degeneration of the retinal pigment epithelium (RPE) in the foveal center causes apoptosis of the photoreceptors and loss of central vision. There is no treatment currently available for GA [3].

In nAMD, choroidal neovascularization (CNV) causes uncontrolled growth of new leaky blood vessels under the macula. This neovascularization is principally driven by the 
vascular endothelial growth factor A (VEGF-A) [3].

The World Health Organization (WHO) has recently highlighted the fact that AMD has become one of the leading causes of severe and irreversible vision loss after cataract and glaucoma $[4,5]$. The global disease burden of AMD is so substantial that it has been listed as a 'Priority Eye Disease' by the WHO, having a global prevalence of $8.7 \%$ [4]. A landmark disease burden progression study on AMD conducted by Wong WL and colleagues in 2014 projected that the global prevalence of AMD would rise to 288 million by $2040[1,6]$.

India too, seems to be reeling under the burden of AMD. The INDEYE study was conducted with an objective to ascertain the age-specific prevalence of early and late AMD in India. Grading of AMD was based on the Wisconsin Age-Related Maculopathy Grading System (WARMGS) validated by the Rotterdam Eye Study. Early AMD was defined as grades 1 to 3 and late AMD as grade 4. The study observed that age specific prevalence of early AMD (grades 1 and 2) was similar to that of Western population, the prevalence of late AMD was $1.2 \%$ in people aged more than 60 years [7]. Another population based cross-sectional study called the Sankara-Nethralaya Rural-Urban Age-Related Macular Degeneration study, conducted in South India, published in 2016, revealed that the prevalence of late AMD was $2.26 \%$ in rural areas as compared to $2.32 \%$ in urban areas [8]. Aging was found to be the most important risk factor in both rural and urban population. This is a cause for concern in developing countries like India, which has an increasing geriatric population [8].

Another retrospective Indian study conducted in a tertiary eye care center found the prevalence of wet AMD to be $2.19 \%$ [9]. This data along with the statistics reveal a demographic shift towards a more aging population and expresses a need for earlier, more accurate diagnosis and initiation of interventional strategies [10]. nAMD is responsible for most of the AMD related blindness [11] and it significantly impacts the functioning, independence and overall quality of life of individuals [12].

Technological and scientific advances have led to ground breaking research in creating effective and safe treatment options like anti-VEGF therapy for patients with nAMD [13]. Three anti-VEGFs viz., ranibizumab (RBZ), aflibercept (AFB) and most recently brolucizumab have been approved for the treatment of nAMD. Bevacizumab (BVZ) is also being used as an off-label treatment strategy $[14,15]$.

Clinical trials for three agents (RBZ, AFB and BVZ) saw approximately $90 \%$ of nAMD patients maintain best corrected visual acuity (BCVA) over 2 years. However, long term studies and real-world settings show significant gaps and challenges from the clinician's perspective, beginning from diagnosis, treatment, monitoring and follow-ups [15].

Clinicians are faced with additional challenges like considerations of delayed diagnosis, individual responses to treatment strategies, sub-optimal long-term therapeutic outcomes, monitoring lapses, financial considerations, lack of compliance, etc. All of these factors add to the complexity of nAMD-related therapeutic decision making and may ultimately lead to disease progression and further vision impairment [15].

This review article will elucidate on the 3 main aspects of dealing with nAMD from the clinician's perspective: Diagnosis, treatment, and follow-up, along with their impact on treatment choices and patient outcomes.

\section{Diagnosis of nAMD: Challenges from a Clinician's Perspective}

There has been substantial evolution with regards to the range of retinal imaging modalities used to diagnose AMD. This can help assess the characteristics of neovascular lesions that guide treatment decisions [16].

Typically, AMD, both globally and in India, is diagnosed based on fundus exam, fundus fluorescein angiography (FFA), color fundus photography, indocyanine green angiography (ICG- A) and optical coherence tomography (OCT) $[9,10]$. Fluorescein angiography is used to confirm the diagnosis and assess the extent of $\mathrm{CNV}$ lesions, leakage and presence of fluid [15].

This section will elaborate on delayed diagnosis and its consequences and challenges associated with OCT imaging.

\subsection{Delayed nAMD: Diagnosis and Its Consequences}

Since $\mathrm{nAMD}$ is a multifactorial disease resulting from the combined contributions of age, genetic predisposition and environmental factors [1, 17], the challenge in diagnosing AMD patients early lies in the fact that patients do not visit a clinician until they are already experiencing symptoms typically associated with intermediate or advanced AMD [10].

Many studies have confirmed that nAMD is under-reported and subsequently remains undiagnosed till symptoms have progressed. Long delays have also been reported between initial symptom diagnosis and initiation of treatment [15]. The mean duration between symptom onset and assessment has been found to be around 2 months, with older patients having longer period of delayed diagnosis. This could be attributed to dependence on caregivers and the common assumption that aging causes gradual vision loss [18].

It is a known fact that $\mathrm{CNV}$ lesions progress rapidly and immature lesions reach maturation within 10 to 14 days, making early diagnosis crucial to maintain vision. Also, most patients remain asymptomatic and may not notice vision loss during this period of lesion growth because the brain compensates the lack of vision. This, when coupled with unawareness about the disease, may be one of the major contributing factors for late presentation [19].

It has been observed in clinical practice, that patients with unilateral nAMD do not pick up early symptoms of vision loss, resulting in delayed diagnosis. On the other hand, bilateral nAMD shows early symptom presentation.

A study conducted by Neely DC and colleagues, published in 2017, revealed underdiagnosis of AMD among 644 people above 60 years of age from Alabama, United States of 
America (USA). A dilated comprehensive eye exam conducted in primary ophthalmology care reported that $25 \%$ of eyes that were deemed to be normal (based the dilated eye examination) had macular degeneration characteristics that were revealed by fundus photography and trained raters. The study also revealed that $30 \%$ of eyes with undiagnosed AMD had large drusen which could have been treated with nutritional supplements, had they been diagnosed earlier [20].

Another study conducted by Parafitt among 621 diagnosed AMD patients from the United Kingdom (UK) revealed that $27 \%$ of patients with nAMD ended up with vision loss due to delayed diagnosis. Reasons for delay in diagnosis as cited by the study included the following: Inability to get an appointment with hospital or optometrist, inability to detect or diagnose AMD at the initial appointment, delayed referrals, delayed communication between specialists [18].

The delays that occur between onset of symptoms and diagnosis can be detrimental and can be attributed to a lack of disease awareness among the general masses and non-specialty health care professionals $[15,21]$.

A study conducted by the National Health and Nutrition Examination Survey (NHANES) published in 2012 showed that $84 \%$ of people from USA with AMD were unaware of their condition [22]. Closer to home, a study conducted by Zhang CX and colleagues among 385 Chinese citizens showed that approximately $70 \%$ of the trial population were not at all familiar with the term AMD. Unawareness of the disease could possibly be one of the reasons for delayed diagnosis [23].

A retrospective study $(n=408$ eyes $)$ conducted in a tertiary center in India revealed that a fourth of the patients presented later in the course of the disease. This impacted treatment initiation and made visual recovery difficult [24].

It can be correlated and safely assumed that delays in diagnosis and treatment initiation of nAMD in the Indian population would be substantially higher as compared to more developed countries like USA or UK. In addition, developing countries like India face shortage of eye care facilities, equipment and experts especially in rural areas which further contribute to delayed diagnosis and timely treatment [25]

\subsection{OCT Imaging}

Unlike other branches of medicine where a biopsy can easily be performed, the practice of vitreoretinal disease is completely dependent on imaging [26]. Being non-invasive, quick and reproducible, OCT is one of the main equipment used for diagnosis of AMD. A correlation between the OCT image and histopathology has enabled almost accurate tissuebased diagnosis [27, 28]. However, the data presented by the OCT devices may be imperfect and may sometimes contain artifacts. Inability to recognize and accurately interpret these artifacts pose a challenge and may lead to faulty diagnosis that can adversely impact the patients [28].

OCT artifacts can be either patient, operator or software related. Patient related artifacts are caused mostly due to eye movements. Eye tracking software can help overcome this. Operator related artifacts include de-centered scans, out of registration due to cut images and degraded images caused due to poor focus. Failed segmentation algorithms resulting in misidentification of inner, outer retinal boundaries, and incomplete segmentation artifacts are software-related. Segmentation failure is another common artifact associated with AMD [27].

Patient and operator related artifacts can be controlled to some extent, but software related artifacts are encountered very often and are difficult to control [27].

The use of OCT as a diagnostic and monitoring tool also presents a few practical challenges in the Indian context viz; the expense incurred due to monthly scans and frequent visits and the dearth of OCT machines, especially in rural areas [25].

\section{Treatment of nAMD: Challenges from a Clinician's Perspective}

Pharmacotherapeutics in the treatment for nAMD have greatly evolved since the introduction of macular photocoagulation in the 1970's. Although pegaptanib was the first anti-VEGF to be approved in 2004 [2], the introduction of RBZ as a successful treatment option for nAMD in 2006 changed both, treatment expectations and outcomes. From then onwards, a number of prospective clinical trials have been conducted to prove time and again the superior VA benefits that can be achieved using anti-VEGFs for nAMD [12]. Many countries who have adopted the treatment of nAMD patients with anti-VEGFs have shown a decrease in legal blindness by almost 50\% [10].

Intravitreally administered anti-VEGF therapy is now the mainstay of AMD treatment since almost 2 decades [15, 29].

Currently, anti-VEGFs are indicated in the first line treatment of nAMD. The most commonly used anti-VEGFs are RBZ, off-label BVZ and AFB [29]. The recently approved brolucizumab has been added to this class of drugs for nAMD. Treating nAMD is associated with many challenges from the clinician's perspective viz., delay in treatment, choice of regimen, lack of sustained vision inter-individual variability and disease reactivation as discussed below.

\subsection{Delay in Treatment Initiation}

It is a well-established fact that once nAMD is diagnosed, treatment should be initiated as soon as possible to prevent further loss of vision. Untreated nAMD can be detrimental and patients have been found to lose up to 4 lines of VA within 2 years of diagnosis, while more than $40 \%$ of diagnosed but untreated patients may lose at least 6 lines by year 3 [30].

Early treatment with anti-VEGFs is indicated especially in patients presenting with CNV. The sub-analyses of MARINA (Minimally Classic/Occult Trial of the Anti-VEGF Antibody Ranibizumab in the Treatment of Neovascular AMD) and ANCHOR (Anti-VEGF Antibody for the Treatment of Predominantly Classic Choroidal Neovascularization in AMD) studies have demonstrated that antiangiogenic treatment leads to better outcomes when early CNV lesion is treated [19].

A retrospective, multi-center epidemiological study 
conducted by Arias et al in Spain showed that the median time from diagnosis to treatment visit was 2.3 months $(95 \% \mathrm{CI}$ : 02-10.8 months). This resulted in a progression of vision loss, demonstrated by a doubling in the number of patients (from $12.4 \%$ to $24.7 \%$ ) who had a visual acuity (VA) of $20 / 400$ or worse [21].

Delays are experienced at many stages of treatment, but the time lapse that seems to occur between diagnosis and treatment initiation is one of the most detrimental since this is the time when the lesions are most active and early treatment initiation may be of great benefit to the patient [21].

Lack of disease awareness, little or no access to specialist eye care may result in delayed visits to clinicians and thereby delayed treatment initiation. On the other hand, inability to manage patients due to stressed clinic capacities may also play a role in the delay of treatment initiation [31].

A cross-sectional study was undertaken by Gilbert $\mathrm{C}$ and colleagues to document existing healthcare infrastructure and practice patterns for managing retinal diseases in India. Data collected from 86 eye units revealed that $60.5 \%$ of eye units managing retinal diseases were located in larger cities revealing a dearth of eye specialty centers in rural areas, where most of the population resides. In addition, India faces shortage of specialized equipment and experts especially in the rural areas, which may ultimately add to the burden of delayed treatment initiation in India [25].

The consequences of delayed treatment can be detrimental and may result in severe vision loss. To prevent this, guidelines recommend treatment for wet AMD to be commenced within 14 days of first presentation or diagnosis $[18,32]$.

A delay between diagnosis and initiation of treatment can be attributed to lack of patient awareness, treatment availability or expert care and reflects the state of healthcare access $[21,23]$.

\subsection{Choice of Regimen}

The pivotal MARINA and ANCHOR trials which used traditional monthly or fixed dose treatment regimens form the basis for the current treatment of nAMD. Monthly fixed dose regimens seem to be the most definitive method of achieving favorable VA gains, but this method is largely impractical and highly burdensome in a clinical practice scenario. Due to this, different treatment regimens like the pro re nata $(\mathrm{PRN})$ and treat and extend $(\mathrm{T} \& \mathrm{E})$ have been introduced and used [33].

PRN is a quarterly regimen where injections are given every 3 months and re-treatment is given only in case of recurrent retinal bleeding or accumulation of fluid. Many trials successfully evaluated the safety and effectiveness of this regimen and showed evidence that PRN treatment regimens, when used in combination with frequent follow-ups, were a viable alternative to the monthly regimen in terms of vision gain and injection burden [33]. Despite its potential value, the PRN regimen still poses a substantial burden on the patient, clinician and the healthcare system [15]. This burden could be attributed to the frequent monitoring required to ascertain the injection frequency.

The quest to balance injection efficacy while maintaining a lower treatment and monitoring burden led to the development of the T\&E regimen [30]. This regimen allowed extension of intervals between injections and has been validated by prospective and retrospective analysis. Studies like TREX-AMD (TReat and EXtend Protocol in Patients with Wet Age-related Macular Degeneration) directly compared monthly dosing to T\&E dosing using RBZ. The results demonstrated a similar vision and anatomic outcome along with a lower treatment burden [15].

Most Western and European countries practice the $\mathrm{T} \& \mathrm{E}$ or treat and observe regimen, the cornerstone of which is progressive lengthening of intervals between the visit-injection dates [14, 34].

This fact was strengthened by the American Society of Retinal Specialists (ASRS) survey conducted over the years. The 2010 survey demonstrated that a majority of clinicians used the PRN regimen (43\%), while $34 \%$ and $17 \%$ used the $\mathrm{T} \& \mathrm{E}$ and monthly regimens respectively. This trend changed by the year 2014 , where most clinicians $(78 \%)$ used a T\&E regimen, while only $2 \%$ of the clinicians still used the monthly regimen and $16 \%$ used the PRN regimen [33].

The results from the Vitreo Retinal Society of India 2017 survey indicated that $40 \%$ of Indian clinicians follow the monthly dosing regimen, with T\&E being used almost equally (39\%). Only $5 \%$ of Indian clinicians used the PRN dosing schedule. (Figure 1) [35].

Most clinicians try to individualize treatment for their nAMD patients by using a combination of initiation (fixed dose for the first 3 months), after which they follow up their patients monthly and treat in case there is exudation (like the PRN strategy) and or use a T\&E method to continue treatment [33].

The chronic, recurring nature of nAMD adds to the limitations of long-term vision gains. Under-treatment, therefore, remains a large unmet need associated with treatment of nAMD [15].

Undertreatment can be ascertained by the number of injections received by patients. It is a known fact that there exists a big difference between the number of injections given to patients in the real world as compared to those in clinical trials [30].

Real world evidence studies do not show as impressive results as those achieved in clinical trials, but satisfactory vision gains can be still achieved. T \& E regimens yield better vision outcomes as compared to PRN even in the real world. A meta-analysis which included 42 real world studies ( $n>26000$ ) demonstrated a greater benefit with the T\&E schedule (Figure 2) [30, 36].

Interestingly, just like the clinical trials, the T\&E group received significantly more injections per year (6.9 vs 4.7; $\mathrm{p}<0.001)$ and had significantly fewer visits (7.6 vs. 8.8; $\mathrm{p}<0.001)$ as compared to the PRN group $[30,36]$. 


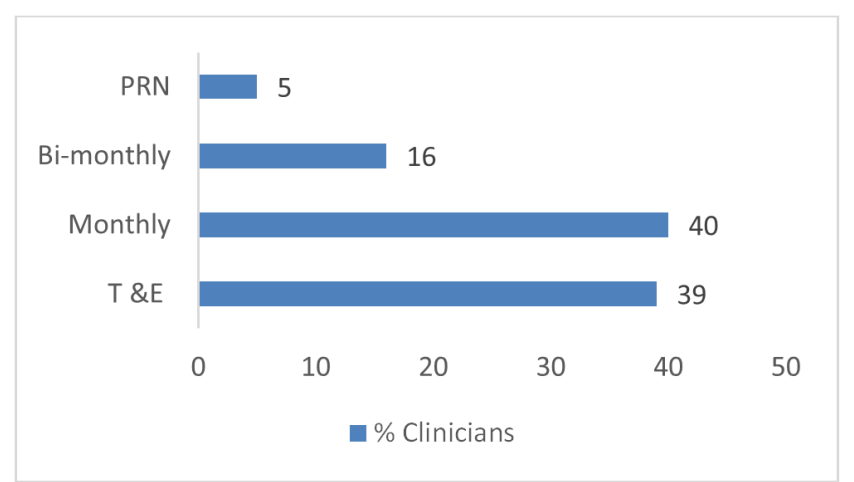

Figure 1. Preferences of treatment regimens (\% clinicians).

T \& E: Treat and extend; PRN: Pro re nata

Adapted from: VRSI Market Research. Final Report. May 2017

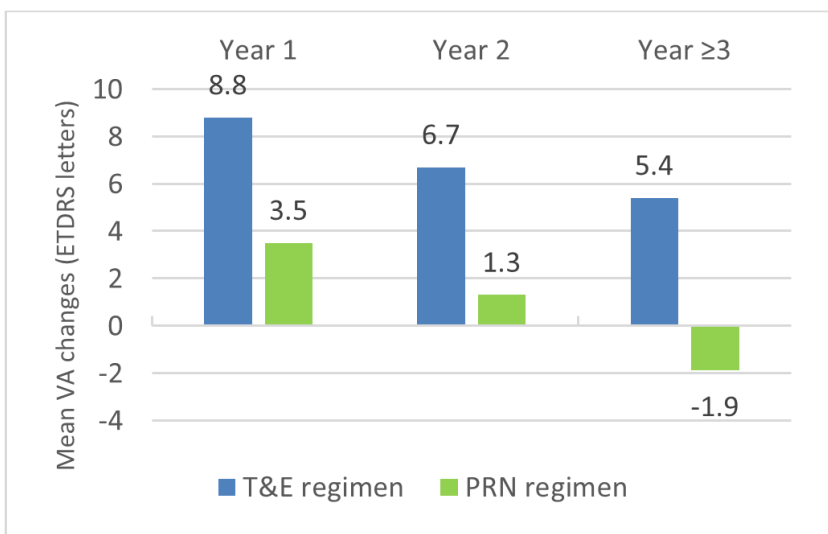

Figure 2. Change in mean $V A \geq 3$ years: Comparison between $T \& E$ and $P R N$ regimens.

T \& E: Treat and extend; PRN: Pro re nata; VA: Visual acuity; ETDRS: Early Treatment Diabetic Retinopathy Study

Adapted from: Mones J, et al. Ophthalmologica 2020; 243: 1-8.

An Indian real-world study conducted by Jain and colleagues followed nAMD patients on anti-VEGFs for up to 1 year. The VA gain was similar between the PRN group and the PRN group with loading dose, with 4.98 injections and 3.7 injections used respectively [14]. However real-world data is sparse in the Indian scenario to have a meaningful conclusion.

Real-world evidence studies suggest that T\&E regimens can be effectively used in clinical practice to achieve vision gains at least for a short duration [30]. However, the low intensity of anti-VEGF treatment in the real world is one of the main factors that accounts for a gradual decline in VA in the long term [30].

The National Institute for Health and Care Excellence (NICE) guidelines 2018 raised concerns about inefficient treatment or under treatment of nAMD in the real-world clinical practice. This could lead inadvertently to discontinuation of treatment in cases where ongoing treatment could be beneficial in improving VA. As a measure to prevent under treatment, NICE provided explicit treatment guidelines prior to determining discontinuation decisions. Such patients should be provided with monitoring guidelines to self-monitor so that treatment can be quickly re-initiated in case of vision deterioration [32].

\subsection{Lack of Sustained Vision}

In the extensions to the key clinical trials, rates of VA maintained or improved in patients treated with anti-VEGFs over the period of around 2 years [15]. However, the chronic nature of nAMD necessitates the treatment to be carried out for more than 2 years [37].

The major studies conducted to evaluate the long-term outcomes were Open-Label Extension Trial of Ranibizumab for Choroidal Neovascularization Secondary to Age-Related Macular Degeneration (HORIZON) and Open-Label Extension Trial of Ranibizumab for Choroidal Neovascularization Secondary to Age-Related Macular Degeneration (SEVEN-UP) [13].

Other studies were the fixed dose interval dosing of anti-VEGF for wet AMD (FIDO) and an observational study by Gilles $\mathrm{M}$, et al, where the divergence in VA outcome between various treatment regimens and how they are sustained over time can be seen (Table 1) [13].

Table 1. Comparison of long-term vision outcomes (Mean letter A).

\begin{tabular}{lllll}
\hline Year & $\begin{array}{l}\text { FIDO } \\
\text { (Mean letter } \boldsymbol{\Delta})\end{array}$ & $\begin{array}{l}\text { SEVEN-UP } \\
\text { (Mean letter } \boldsymbol{\Delta} \text { ) }\end{array}$ & $\begin{array}{l}\text { HORIZON } \\
\text { (Mean letter } \boldsymbol{\Delta} \text { ) }\end{array}$ & $\begin{array}{l}\text { Gillies } \\
\text { (Mean letter } \boldsymbol{\Delta} \text { ) }\end{array}$ \\
\hline 0 & 0.0 & 0.0 & 0.0 & 0.0 \\
1 & 13.2 & & & 5.0 \\
2 & 16.1 & 11.2 & 9.0 & \\
3 & 15.4 & & 4.1 & 2.2 \\
4 & 14.6 & 1.7 & 2.0 & \\
5 & 14.0 & & -0.1 & 0.7 \\
6 & 12.2 & & & \\
7 & 12.1 & -8.6 & & -2.7 \\
\hline
\end{tabular}

Mean letter $\Delta$ was calculated from baseline. HORIZON was an extension trial that followed patients existing from ANCHOR and MARINA trials after two years of monthly therapy. The SEVEN-UP study was a further 3-years extension of the patients exiting the HORIZON trial. FIDO: Fixed dose interval dosing of anti-VEGF for wet AMD; SEVEN-UP: Fellow Eye Comparisons for 7-Year Outcomes in Ranibizumab-Treated AMD Subjects From ANCHOR, MARINA, and HORIZON; HORIZON: An Open-Label Extension Trial of Ranibizumab for Choroidal Neovascularization Secondary to Age-Related Macular Degeneration.

Adapted from: Peden $\mathrm{MC}$ and Suner IJ. https://www.retina-specialist.com/article/answers-to-the-three-big-questions-of-longterm-treatment-of-wet-amd Accessed on 13 Feb2020

The study reported the 7-year outcome of treatment naive patients who received anti-VEGF therapy $(n=131$ eyes). It also demonstrated that $40 \%$ patients maintained their vision (20/40) at 7 years, but there was also a 2.7- letter loss from baseline. This data was comparable to the CATT 5-year data, which reported a 3-letter loss from baseline at 5 years [13].

In the HORIZON and Comparison of Age-related Macular Degeneration Treatments Trials (CATT), the injection frequency after the initial loading doses was decided by the attending clinician and they included both monthly and PRN dosing, resulting in a reduction in the number of injections in the PRN group compared to the fixed dose group and an associated decline in VA [13]. When treatment frequency was evaluated in the CATT trial, it was observed that $71 \%$ of 
patients who were followed up to 5.5 years only received 4.8 injections in year 3, 4.5 injections in year 4 and 4 injections in year 5. The results of the CATT study therefore showed a sustained decline in VA over time, which could be attributed to a fewer number of injections or under treatment [30].

On the other hand, the FIDO study showed a VA gain of 12.1 letters from baseline to 7 years if the patient continuously received a fixed-interval dosing between 4 to 8 weeks, with the mean number of injections received being 10.5 per year. Overall, the analysis of the long-term effect of anti-VEGFs revealed that more the number of injections, higher were the chances of maintenance or improvement of vision [13].

However, achieving optimal long-term treatment outcomes in the real world can prove to be challenging. A real-world analysis carried out over 5 years on 1083 nAMD patients on PRN treatment revealed that initial VA gain was not maintained over 5 years. In fact, $34 \%$ of patients experienced loss of $>15$ letters. The injection frequency reduced from 6 in the first year to between 4 and 5 in the rest of the years [30, 38].

Another long-term analysis from the Fight Retinal Blindness (FRB) registry where 1212 eyes were treated monthly with RBZ, showed that maximum VA gains were observed at month $6(+6.3$ letters). By the end of 5 years, the VA returned to baseline $(+0.7$ letters $)$ and in the eyes that were still followed till 7 years $(n=131)$, the VA dropped below the baseline $(-2.7$ letters). The median number of injections was 6 in the first year. The number of injections between year 2 to year five were 5 (4.9; SD, 3.1), 5 (4.9; SD, 3.5), 6 (5.4; SD, 3.3) and 5 (4.9; SD; 3.3) respectively [39].

A single center, retrospective real-world study using AFB in $\mathrm{nAMD}$ patients was conducted by Traine $\mathrm{P}$, et al $(\mathrm{n}=231$ eyes) and followed till year 4 to ascertain the visual outcomes of patients using the $\mathrm{T} \& \mathrm{E}$ dosing regimen. The results demonstrated an increase in mean BCVA from 59.8 letters at baseline to 65.8 letters after the loading dose, to 65.5 letters at year 1 . The VA at 4 years was higher than that at baseline but the difference was not significant (63.4 letters). These results were achieved with an injection frequency of 7.7 at year 1 and 4.4 injections per year during the second to fourth year of follow-up [40].

Regimens used in clinical trials are often not achievable in real world settings as demonstrated by numerous studies mentioned above.

Factors that underlie the reduced effectiveness of anti-VEGFs in clinical practice are as follows:

1. Reduced number of injections: It is not surprising to note that the number of injections is much lower in real world studies than in clinical trials. This can be attributed to the lack of adherence to intensive treatment regimens [41, 42].

2. Schedule or drug regimen and monitoring: Typical PRN regimens do not reduce the burden of monitoring, leading to lack of adherence to treatment. Also, PRN treatment, by definition, only treats patients with symptomatic disease. In these cases, recurring fluid can lead to progressive damage and worsening VA. The $\mathrm{T} \& \mathrm{E}$ regimen has emerged as a feasible alternative to the PRN dosing and is being increasingly used by clinicians [41].

3. Worse baseline VA: Studies have demonstrated that patients with good baseline VA demonstrated smaller VA changes as compared to those with poor baseline VA, while treatment-naïve patients were observed to have better outcomes than those individuals who were previously treated [41].

It is important that the clinician understands that there seems to be no 'one size fits all' approach on the right anti-VEGF regimen to manage nAMD and focuses on individualizing therapy so that patients can gain maximum benefits from anti-VEGF therapy.

The perspective of the physician in this scenario is well illustrated by the 2018 ASRS Preferences and Trends (PAT) membership survey which involved around 1029 retina specialists from all over the world. Approximately $70 \%$ of the retina specialists from the Asia Pacific region felt that there was a requirement for long acting or sustained delivery therapy [30, 43].

Almost $43 \%$ of global retina specialists surveyed treat fewer than $10 \%$ of their nAMD patients at 4-week intervals for an extended period of time and $>50 \%$ would consider a product that would maintain their patients with a long-term efficacy [30].

Closer to home, a survey conducted by the Vitreoretinal Society of India (VRSI) in 2017 which covered 113 clinicians from 24 locations around India revealed that $84 \%$ of wet AMD patients were treated with anti-VEGFs, with almost $40 \%$ adapting the T\&E method and $48 \%$ still following the monthly regimen. Number of injections reduced as the years progressed. (Figure 3) [35].

The successful management of nAMD with anti-VEGFs have acted as an opportunity for development of more effective drugs that overcome these unmet needs and work over a longer period. These will ensure fewer injections and hence better compliance. Brolucizumab is one such newly approved anti-VEGF. Other drugs in the pipeline include faricimab, the RBZ port delivery system, etc. [44].

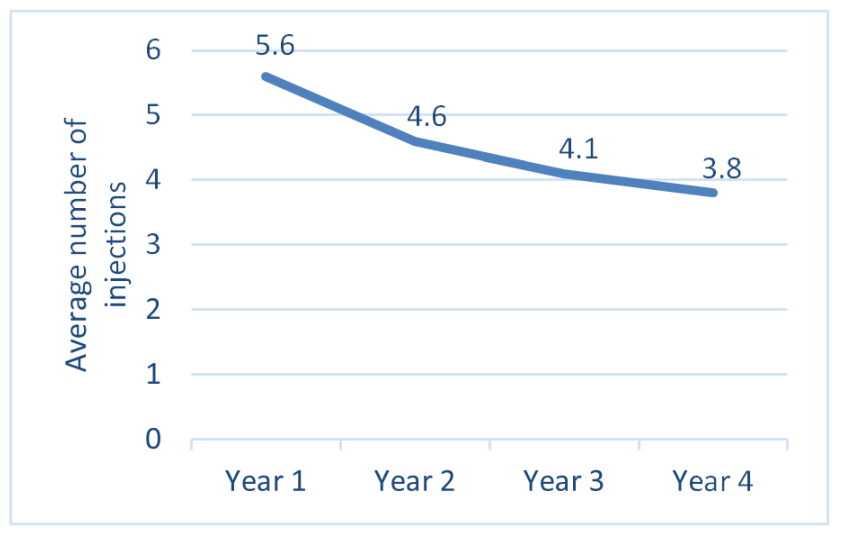

Adapted from: VRSI Market Research. Final Report. May 2017

Figure 3. Average number of anti-VEGF injections. 


\subsection{Inter-individual Variability Leading to Under-treatment}

Although anti-VEGFs are very effective in improving VA in nAMD patients, a major unmet need is that many patients do not gain vision even with maximum therapy [45]. This could be attributed to the variable nature of nAMD. It is important to understand that nAMD is a disease that encompasses a large spectrum of pathogenic factors, genetic backgrounds, phenotypic features and individualized responses to therapy. Many studies which assessed PRN regimens have pointed out the inter-individual variability with respect to the need for anti-VEGF therapy. The downside to individualized PRN regimens is the constant uncertainty as to when the patient would need the next injection and the constant monitoring that would be required to ascertain that need [34].

Although the management of the disease is very individualistic, it is important to optimize therapeutic strategies by altering the injection frequency; which in turn is an important determinant of patient outcomes [15].

\subsection{Disease reactivation}

Another important nAMD related challenge is the unpredictability of the disease, viz., disease recurrence and consequently vision loss after treatment is discontinued. The causes for reactivation could range from foveal atrophy, growth of fibrotic scars and recurrences of $\mathrm{CNV}$, but the consequence of reactivation of nAMD is irreversible blindness which is unacceptable [37].

Unpredictability may contribute towards lack of sustained efficacy or sub-optimal outcomes of any treatment regimen $[15,37]$. A study conducted by Nguyen et al, reported that just after 1 year of treatment suspension, $41 \%$ eyes demonstrated reactivation, the number increasing to $79 \%$ at 5 years. The study also demonstrated that among the eyes which showed reactivation of lesions there was a sustained loss of 4.2 letters, of which only 1.2 letters could be recovered after treatment commencement. The study reported that VA better than 20/40 at the time of treatment suspension and a short treatment duration of approximately 3 years were associated with greater risk of reactivation. This study proved that treatment suspension is dangerous due to higher risk of recurrence that may lead to irreversible vision loss [37].

Disease reactivation is an undeniable factor that needs to be considered before treatment suspension because the burden of irreversible vision loss can be a greater burden that ongoing treatment [37].

\section{Follow-up of nAMD Treatment: Clinician's Perspective}

nAMD needs to be frequently evaluated either to decide on the next dose (if $\mathrm{T} \& \mathrm{E}$ or PRN regimens are used) or to monitor disease activity [46] especially during the first two years of treatment [47].
The American Association of Ophthalmology Preferred Practice Patterns (AAO PPP) 2019 guideline recommends that patients should be regularly tested for disease progression using OCT, OCTA or FA. Follow-up intervals could be based on the discretion of the practicing clinician [1].

This section deals with challenges faced by clinicians after treatment is initiated and includes burden of monitoring, cost of continued therapy, challenges encountered by the clinician during counselling, referrals, managing persistent cases and rehabilitation.

\subsection{Burden of Monitoring Visits}

Although recommended by guidelines, indefinite evaluation, monitoring visits, time spent on routine monitoring and costs involved certainly poses a huge burden in the real-world setting, impacting monitoring and in turn leading to a commensurate decrease in VA of many patients [48].

Monitoring visits contribute largely to under treatment as they consume a huge amount of time and resources, burdening the already limited personnel (clinicians and technicians), machines (like OCT) and examination space. These resource burdens in turn lead to under treatment of nAMD and can be considered as a major unmet need in this space [34].

A multi-center survey conducted in USA among 57 retina specialists reported that an average total of 90 minutes (range 13 minutes to $>4$ hours) was spent on care provided to nAMD patients per visit, which accounted for almost $20 \%$ of the time of the office staff per week. It was interesting to note that $58 \%$ of the clinicians felt that billing and filing for reimbursement places a major burden on staff resources [15, 49]. A similar study conducted in Spain reported that shortage of staff and facility issues were main limitations for managing nAMD patients [46].

A retina specialist survey conducted in the United States of America reported that on an average 23 staff members were involved in the care of nAMD patients, with technicians and billing personnel spending more than $20 \%$ of their time on nAMD related care giving. New patients spend more time with technicians and photographers during initial visits and require a longer duration for examination by the clinician. Figure 4 elucidates the flow of patients through various parts of their visit and the time spent on various aspects of their appointment [49].

It is interesting to note that majority of the retina specialists $(62 \%)$ felt that patient monitoring placed a significant burden on staff time. Two thirds of the clinicians stated that reduction in office visits would be desirable [49].

All the studies demonstrate that practices are highly invested in managing nAMD patients and struggle to maintain a balance between quality of care and resource-related expenses [49].

The 2018 Preferences and Trends Survey conducted by ASRS revealed that $66 \%$ of the clinicians felt the treatment burden is one of the great unmet needs from the nAMD treatment perspective $[30,43]$. 
The relevant data from an Indian perspective on the amount of time spent and the resources involved in management of nAMD patients is lacking, however it can be assumed to be on similar lines or even greater. However, there is a need for Indian data in this aspect.

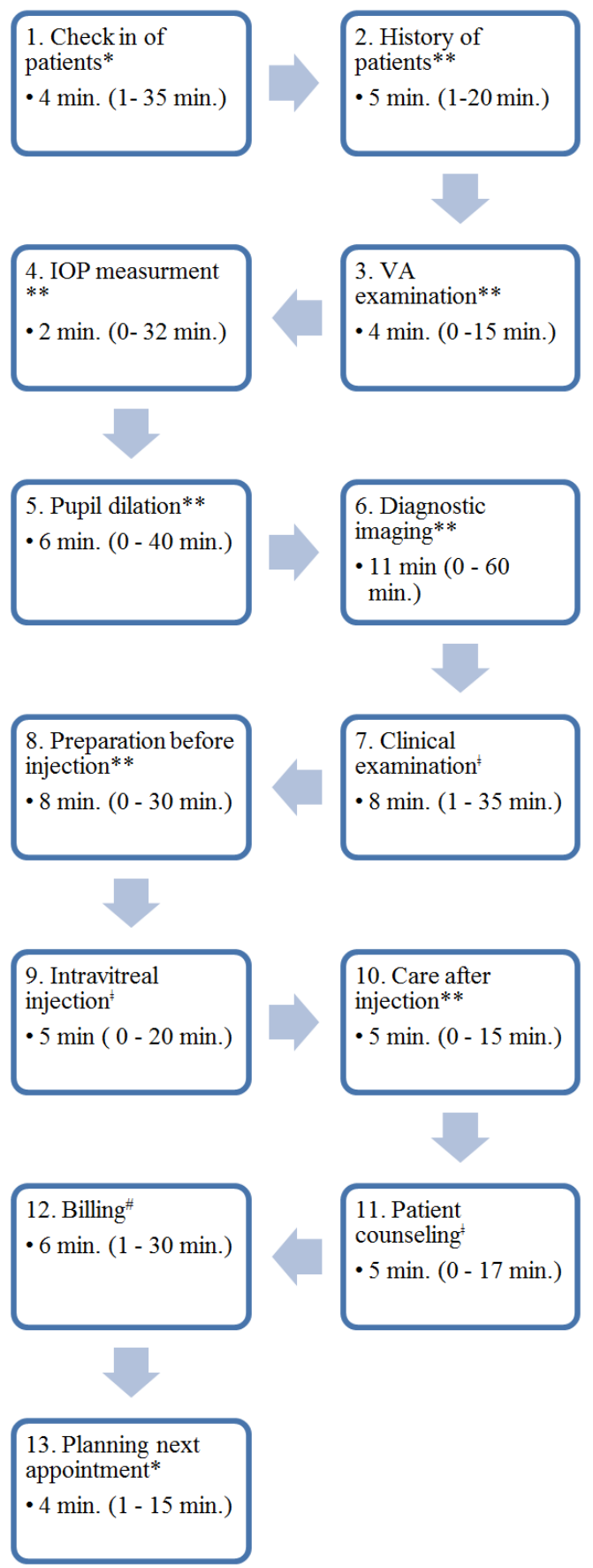

Figure 4. Series of steps involved in the management of $n A M D$ patients and average time range per step.

Cumulative patient wait time: $34 \mathrm{~min} .(0-142 \mathrm{~min}.) \mathrm{N}=221$.

Average Total: 90 min. (Range $13-261$ min., median 85)

*Receptionist; **Technician; 'Retina specialist; ${ }^{*}$ Office manager

Average times will not be sum of average total minutes due to differences in range for each step in the patient flow

IOP: Intraocular pressure; VA: Visual acuity

Adapted from: Prenner JL et al. Am J Ophthalmol 2015; 160 (4): 725-731.

\subsection{Clinic Services and Personnel}

Increases in the numbers of nAMD patients are expected to continue and can be attributed to the chronic nature of the disease and an ageing population. This puts a great burden on the already stressed clinic capacity and clinic services like patient counselling. Inability to handle the clinic load, lack of or burdened clinic staff, dearth of equipment and technicians can threaten optimal care and reduce access to the potential sight saving treatment for nAMD patients [50].

\subsubsection{Patient Counselling}

Counselling is an important service offered in the clinic. It forms an important part of anti-VEGF therapy, but it is also a time-consuming process placing strain on busy clinic and personnel schedules. However, counselling is imperative especially in patients with advanced disease or for those who are unresponsive to anti-VEGF treatment. Counselling sessions involve explaining to patients and their families the need for intravitreal injections, the importance of complying to therapy, the consequences of non-compliance and cost of treatment [48].

The AAO PPP 2019 for AMD recommends educating patients about the disease prognosis, the potential value of treatment, reassurance about intravitreal therapy and cessation of smoking. A discussion on the risks and benefits of treatment and treatment alternatives is recommended [1]. Counselling should aim to encourage and support patients to self-monitor their vision on a daily basis [32].

Counselling should be such that it enables patients with nAMD to report new symptoms or changes like blurred or grey patch in their vision, straight lines appearing distorted and objects appearing smaller than normal.

Counselling however, increases the interaction time between clinician and patients, puts additional burden on stressed clinic capacities and may also involve hiring of trained resources.

\subsubsection{Equipment}

Clinics that cater to an increasing number of patients may also face a dearth of equipment. The equipment cost may also be a significant factor in their availability.

A multi-city survey conducted in India by Gilbert et al demonstrated that facilities for FFA and OCT were significantly higher in stand-alone eye hospitals as compared to multi-specialty hospitals $(\mathrm{P}=0.02)$ and in teaching facilities $(\mathrm{P}=0.001)$ as compared to non-teaching facilities [25].

\subsubsection{Trained Personnel}

A dearth of trained personnel may prove catastrophic in terms of diagnosis as well as routine management. Clinics are severely burdened by lack of trained personnel like counsellors, nurses and technicians.

A cross sectional study which was conducted in 11 cities across India including 64 facilities showed that eye units in larger cities and those that are privately funded had a higher number of retina specialists. Many of the facilities had nurses 
trained in ophthalmology, but there seemed to be a dearth in staff qualified for low vision care and counsellors (4 out of 10 facilities). Only $1 / 3 \mathrm{rd}$ of the facilities had a trained retinal photographer [25].

This study reinforces the fact that India faces a dearth of qualified personnel who can deal with patients on nAMD treatment as well as the necessary equipment needed to diagnose and monitor treatment progress. This can lead to gross under treatment and ultimately vision loss [25].

There is a need to identify the clinic and personnel related burden and increase awareness of their consequences in a bid to ensure that clinical services adapt to the growing needs of the nAMD patients and provide fast and efficient services. Overcoming the clinic and personnel burden may prove beneficial to diagnose and monitor patients afflicted with nAMD. Key capacity issue includes lack of clinic space considering the increasing number of nAMD patients. Shortage of staff including retinal consultants and medical retinal staff may lead to increased patient waiting time and impact on clinical efficiency. [50]. Lack of equipment, time used for caring for nAMD patients, the monitoring visits etc., together place a huge burden on the already burdened health care systems and there is an urgent need to consider all of these factors to optimize nAMD treatment.

\section{Way Ahead for Treating and Monitoring of nAMD}

Considering the evidence from pivotal trials and real-world evidence, it can be safely assumed that there seem to be many challenges in the management, monitoring and follow-ups of nAMD patients. To make matters worse, the disease is chronic, highly variable and unpredictable leaving clinicians grappling with undertreated patients and other infrastructure associated burdens. Although guidelines have been created to ease the management pathways of nAMD, the treatment strategies have to be highly individualistic keeping in mind financial burden on the patient and infrastructure burden and resource involvement.

The Vision Academy Steering Committee is a global group of ophthalmic experts proposed 4 key principles to identify and address the unmet needs in anti-VEGF management [51]:

1. Maximize and maintain VA benefits for all patients.

2. Decide when to treat next, rather than whether to treat now.

3. Titrate the treatment intervals to match patients' needs.

4. Treat at each monitoring visit.

Adopting a more personalized approach and reduced treatment burden will help improve patient compliance.

Anti-VEGFs have revolutionized the treatment of nAMD, but several unmet needs still continue. To address these unmet needs, several new treatment options are in the pipeline. A longer acting anti-VEGF, brolucizumab has already been approved by the USFDA [47].

Newer molecules and sustained-delivery systems which can target novel angiogenesis check points are also being developed. Therapies like NT-503 vitreous implant are being researched to ensure sustained delivery. These delivery systems are being developed with a hope to address the treatment burden caused by frequent injections. Other such sustained delivery implants in development are the RBZ port delivery system and the posterior micropump drug delivery system. [47].

Gene therapies are also being developed to influence the host cells to produce proteins that bind to VEGF. These promise long-term durable results after just 1 administration [47].

\section{Conclusion}

nAMD is a rapidly progressing degenerative eye disease, that has the potential to cause irreversible vision loss. Although anti-VEGF treatments have improved outcomes, the clinician is still faced with many unmet treatment-related challenges such as delayed presentation and diagnosis, different treatment regimes, lack of sustained vision gains, inter-individual variability and the chronic nature of the disease. In addition, the clinical workload that is associated with frequent monitoring places additional burdens. These can be attributed to a combination of a large patient population, stressed clinic capacities, lack of personnel and equipment. Longer acting anti-VEGFs may help lighten this burden as they can potentially increase the duration between two injections without compromising the VA. This in turn will reduce the burden on the already stressed out clinic capacity and personnel, thus providing better quality care for all.

There is an urgent need to highlight these treatment and follow-up related challenges, especially in developing countries like India where clinicians manage high patient numbers with strained health care resources. There is a severe dearth of India-specific studies on the challenges faced by clinicians while treating patients with nAMD. These studies can act as a basis to form effective strategies to manage patients with $\mathrm{nAMD}$ and to ensure that they receive optimal care and have access to potentially sight saving treatment.

\section{Acknowledgements}

Medical writing support in the development of this paper was provided by Mool Tatvam Consulting LLP.

\section{References}

[1] American Academy of Ophthalmology. Age-related macular degeneration preferred practice pattern. 2019. Available [Online] at URL: https://www.aao.org/preferred-practice-pattern/age-related-ma cular-degeneration-ppp Accessed on 6th Feb 2020.

[2] Al-Zamil WM, Yassin SA. Recent developments in age-related macular degeneration: a review. Clinical Interventions in Aging 2017; 12: 1313-30. 
[3] Holekamp NM. Review of Neovascular Age-Related Macular Degeneration Treatment Options Am J Manag Care 2019 Jul; 25 (10 Suppl): S172-S181.

[4] WHO. Causes of blindness and visual impairment. Available [Online] at: http://www.who.int/blindness/causes/en/ Accessed on 4 October 2018

[5] Gheorghe A, Mahdi L, Musat O. Age related macular degeneration. Rom J Ophthalmol. 2015; 59: 74-7.

[6] Wong WL, Su X, Li X, Cheung CM, Klein R, Cheng CY, et al. Global prevalence of age-related macular degeneration and disease burden projection for 2020 and 2040: a systematic review and meta-analysis. The Lancet Global Health. 2014 Feb 1; 2 (2): e106-16.

[7] Krishnan T, Ravindran RD, Murthy GV, Vashist P, Fitzpatrick $\mathrm{KE}$, Thulasiraj $\mathrm{RD}$, et al. Prevalence of early and late age-related macular degeneration in India: the INDEYE study. Investigative ophthalmology \& visual science. $2010 \mathrm{Feb} 1 ; 51$ (2): 701-7.

[8] Raman R, Pal SS, Ganesan S, Gella L, Vaitheeswaran K, Sharma T. The prevalence and risk factors for age-related macular degeneration in rural-urban India, Sankara Nethralaya Rural-Urban Age-related Macular degeneration study, Report No. 1. Eye 2016; 30: 688-97.

[9] Gopalakrishnan S, Velu S, Raman R. Low-vision intervention in individuals with age-related macular degeneration. Indian J Ophthalmol 2020; 68: 886-9.

[10] Bandello F. Age-Related Macular Degeneration. Early detection and intervention. An Unmet Need in Age-Related Macular Degeneration. Available [Online] at: https://www.maculogix.com/wp-content/uploads/An-Unmet-N eed-in-Age-Related-Macular-Degeneration.pdf Accessed 8th December 2019.

[11] Jaffe DH, Chan W, Bezlyak V, Skelly A. The economic and humanistic burden of patients in receipt of current available therapies for nAMD. Journal of comparative effectiveness research. 2018 Nov; 7 (11): 1125-32.

[12] Agarwal A, Aggarwal K, Gupta V. Management of neovascular Age-related macular degeneration: A review on landmark randomized controlled trials. Middle East Afr J Ophthalmol 2016; 23: 27-37.

[13] Peden MC and Suñer IJ. Answers to the Three Big Questions of Long-Term Treatment of Wet AMD. Retina Specialist. Available [Online] https://www.retina-specialist.com/article/answers-to-the-threebig-questions--of-longterm-treatment-of-wet-amd Accessed on 13 Feb 2020 .

[14] Jain N, Yadav NK, Jayadev C, Srinivasan P, Mohan A and Shetty BK. The ARMOUR Study: Anti-VEGF in Neovascular AMD - Our Understanding in a Real-World Indian Setting. Asia-Pac J Ophthalmol 2017; 6: 488-492.

[15] Wycoff CC, Clark WL, Nielson JS, Brill JV, Greene LS and Heggen CL. Optimizing Anti-VEGF Treatment Outcomes for Patients with Neovascular Age-Related Macular Degeneration. JJ Manag Care Spec Pharm. 2018; 24 (2-a): S3-S15.

[16] Freund KB, Toth $\mathrm{C}$ and Zarbin M. Best Clinical Practice for Age-Related Macular Degeneration Imaging Journal of Vitreo Retinal Diseases 2019. DOI: 10.1177/2474126419834702.
[17] Volz C and Pauly D. Antibody therapies and their challenges in the treatment of age related macular degeneration. European Journal of Pharmaceutics and Biopharmaceutics 2015; 95: $158-172$.

[18] Parafitt A, Boxell E, Amoaku WM and Bradley C. Patient-reported reasons for delay in diagnosis of age-related macular degeneration: a national survey. BMJ Open $\begin{array}{llll}\text { Ophthalmology 2019; 4: e000276. doi: } & \end{array}$ 10.1136/bmjophth-2019-000276.

[19] Schwartz R and Loewenstein A. Early detection of age-related macular degeneration: Current status. Int J Retin Vitr (2015) 1: 20 DOI 10.1186/s40942-015-0022-7.

[20] Neely DC, Bray KJ, Huisingh CE, Clark ME, McGwin Jr G and Owsley C. Prevalence of undiagnosed age-related macular degeneration in primary eye care. JAMA Ophthalmol. 2017 Jun; 135 (6): $570-575$.

[21] Arias L, Armada' F, Donate J, Garc1'a-Arumı' J, Giralt J, Pazos $\mathrm{B}$, et al. Delay in treating age-related macular degeneration in Spain is associated with progressive vision loss. Eye 2009; 23: 326-333.

[22] Gibson D. Diabetic retinopathy and age-related macular degeneration in the US. Am J Prev Med 2012; 43 (1): 48-54.

[23] Zhang CX, Zhang GM, Ma N, Xia S, Yang JY, Chen YX. Awareness of age-related macular degeneration and its risk factors among beijing residents in China. Chinese medical journal. 2017 Jan 20; 130 (2): 155.

[24] Sudhalkar A, Sethi V, Gogte P, Bondalapati S, Khodani M, Chhablani JK. Retrospective hospital-based analysis of age-related macular degeneration patterns in India: 5-year follow-up. Indian journal of ophthalmology. 2015 Dec; 63 (12): 899.

[25] Gilbert C, Babu GR, Gudlavalleti AS, Anchala R, Shukla R, Ballabh $\mathrm{PH}$, et al. Eye care infrastructure and human resources for managing diabetic retinopathy in India: The India 11-city 9-state study. Indian J Endocrinol Metab. 2016 Apr; 20 (Suppl 1): $\mathrm{S} 3-\mathrm{S} 10$

[26] Staurenghi Giovanni, Sadda S, Chakravarthy U and Spaide RF Proposed lexicon for anatomic landmarks in normal posterior segment spectral-domain optical coherence tomography. The IN.OCT consensus. Ophthalmology 2014; 1-7. DOI: 10.1016/j.ophtha.2014.02.023.

[27] Bhende M, Shetty S, Parthasarathy MK and Ramya S. Optical coherence tomography: A guide to interpretation of common macular diseases. Indian J Ophthalmol 2018; 66: 20-35.

[28] Chen TC. Don't be fooled. Spotting OCT defects. Review of ophthalmology. Available [Online] at: https://www.reviewofophthalmology.com/article/dont-be-fool ed-spotting-oct-artifacts Accessed on 5 May 2020.

[29] Schlottmann PG, Alezzandrini AA, Zas M, Rodriguez FJ, Luna $\mathrm{JD}$ and $\mathrm{Wu} \mathrm{L}$. New Treatment Modalities for Neovascular Age-Related Macular Degeneration. Asia-Pac J Ophthalmol 2017; 6: 514-519.

[30] Mones J, Singh RP, Bandello F, Souied E, Liu X and Gale R. Undertreatment of Neovascular Age-Related Macular Degeneration after 10 Years of Anti-Vascular Endothelial Growth Factor Therapy in the Real World: The Need for A Change of Mindset. Ophthalmologica 2020; 243: 1-8. 
[31] Ly A, Nivison-Smith L, Hennessy $M$ and Kalloniatis $M$. Collaborative care of non-urgent macular disease: a study of inter-optometric referrals. Ophthalmic Physiol Opt 2016, 36: 632-642.

[32] National Institute for Health and Care Excellence 2018. Age-related macular degeneration: diagnosis and management. NICE Guideline NG82 January 2018. Available [Online] at: https://www.nice.org.uk/guidance/ng82 Accessed on 5th May 2020.

[33] Patel AV, Barb SM and Young LH. Finding the Optimal Treatment Plan for Exudative AMD. Medscape. Available [Online]

https://www.medscape.com/viewarticle/851042_print

Accessed on 11 August 2020.

[34] Mantel I. Optimizing the anti-VEGF treatment strategy for neovascular age-related macular degeneration: from clinical trials to real-life requirements. Trans Vis Sci Tech. 2015; 4 (3): 6, doi: 10.1167/tvst.4.3.6.

[35] VRSI Market Research Final Report. May 2017. Available [Online] at: http://vrsi.in/wp-content/uploads/2017/07/VRSI-Market-Resea rch.pdf Accessed on 30th June 2020.

[36] Kim LN, Mehta H, Barthelmes D, Nguyen V, Gillies MC. Metaanalysis of real-world outcomes of intravitreal ranibizumab for the treatment of neovascular age-related macular degeneration. Retina. 2016 Aug; 36 (8): 1418-31.

[37] Khurana RN. Long-Term Management of Neovascular Age-Related Macular Degeneration: To Suspend or Not to Suspend? American Academy of Ophthalmology Editorial 2019. DOI: $10.1016 /$ j.oret.2019.04.013.

[38] Wecker T, Ehlken C, Bühler A, Lange C, Agostini H, Böhringer $\mathrm{D}$, et al. Five-year visual acuity outcomes and injection patterns in patients with pro-re-nata treatments for AMD, DME, RVO and myopic CNV. Br J Ophthalmol. 2017 Mar; 101 (3): 353-9.

[39] Gillies MC, Campain A, Barthelmes D, Simpson JM, Arnold JJ, Guymer RH, et al on behalf of the Fight Retinal Blindness Study Group. Long-Term Outcomes of Treatment of Neovascular Age-Related Macular Degeneration. Ophthalmology September 2015; 122 (9): 1837-1845.

[40] Traine PG, Pfister IB, Zandi S, Spindler J, Garweg JG. Long-term outcome of intravitreal aflibercept treatment for neovascular age-related macular degeneration using a "treat-and-extend" regimen. Ophthalmology Retina. 2019 May $1 ; 3(5): 393-9$.

[41] Chong V. Ranibizumab for the treatment of wet AMD: a summary of real-world studies. Eye. 2016 Feb; 30 (2): 270-86.
[42] Holz FG, Tadayoni R, Beatty S, Berger A, Cereda MG, Cortez $\mathrm{R}$, et al. Multi-country real-life experience of anti-vascular endothelial growth factor therapy for wet age-related macular degeneration. Br J Ophthalmol 2015; 99: 220-226.

[43] ASRS global trends in retina. 2018 Available [Online] at: https://www.asrs.org/content/documents/2018-global-trends-in -retina-survey-highlights-website.pdf Accessed on 1st May 2020.

[44] Singer $M$ and Rahman EZ. Update on Longer-Acting Anti-VEGF Therapies. An expansive horizon is coming into focus. Retinal Physician, Volume: 17, Issue: April 2020, page(s): $38-41,49$.

[45] Slakter J. Unmet needs in wet AMD. MD mag. Available [Online] at URL: https://www.mdmag.com/conference-coverage/aao-2017/jason -slakter-md-unmet-needs-in-wet-amd Accessed on $10 \mathrm{Dec}$ 2019.

[46] Garc'ia-Layana A, Garc'ia-Arum'I J, Figueroa MS, Barquet LA, Ru' 1z-Moreno JM, Moncl 'us-Arbona L, and The Spanish AMD Multicenter Group. Management of Wet Age-Related Macular Degeneration in Spain: Challenges for Treat and Extend Implementation in Routine Clinical Practice. Journal of Ophthalmology. Volume 2019, Article ID 9821509. DOI: $10.1155 / 2019 / 9821509$.

[47] Klufas MA and D'Amico DJ. Targeting Unmet Needs in nAMD Treatment. Retina specialist. Available [Online] at: https://www.retina-specialist.com/article/targeting-unmet-need s-in-namd-treatment Accessed on 27th November 2019.

[48] McKinney S. Easing the Anti-VEGF Treatment Burden. Review of ophthalmology. January 2020. Available [Online] at: https://www.reviewofophthalmology.com/article/easing-the-an tivegf-treatment-burden Accessed on 10th May 2020.

[49] Prenner JL, Halperin LS, Rycroft C, Hogue S, Liu ZW and Seibert R. Disease Burden in the Treatment of Age-Related Macular Degeneration: Findings From a Time-and-Motion Study. Am J Ophthalmol 2015; 160 (4): 725-731.

[50] Amoaku W, Blakeney S, Freeman M, Gale R, Johnston R, McLaughlan B, et al. Action on AMD. Optimising patient management: act now to ensure current and continual delivery of best possible patient care. Eye (Lond). 2012 Feb; 26 (Suppl 1): $\mathrm{S} 2-\mathrm{S} 21$

[51] Lanzetta P, Loewenstein A, Vision Academy Steering Committee. Fundamental principles of an anti-VEGF treatment regimen: optimal application of intravitreal anti-vascular endothelial growth factor therapy of macular diseases. Graefe's Archive for Clinical and Experimental Ophthalmology. 2017 Jul 1; 255 (7): 1259-73. 